DOI: https://doi.org/10.24297/jam.v16i0.8152

\title{
A New Inexact Non-Interior Continuation Algorithm for Second-Order Cone Programming
}

\author{
Liang Fang \\ College of Mathematics and Statistics, Taishan University, 271000, Tai'an, China \\ Email: fangliang3@163.com
}

\begin{abstract}
Second-order cone programming has received considerable attention in the past decades because of its wide range of applications. Non-interior continuation method is one of the most popular and efficient methods for solving second-order cone programming partially due to its superior numerical performances. In this paper, a new smoothing form of the well-known Fischer-Burmeister function is given. Based on the new smoothing function, an inexact non-interior continuation algorithm is proposed. Attractively, the new algorithm can start from an arbitrary point, and it solves only one system of linear equations inexactly and performs only one line search at each iteration. Moreover, under a mild assumption, the new algorithm has a globally linear and locally Q-quadratical convergence. Finally, some preliminary numerical results are reported which show the effectiveness of the presented algorithm.
\end{abstract}

Keywords: Second-order cone programming; non-interior continuation method; interior-point method; smoothing function; Euclidean Jordan algebra.

\section{Introduction}

Second-order cone programming (SOCP for simplicity) is convex optimization in which a linear function is minimized over the intersection of an affine linear manifold with the Cartesian product of several second-order cones. SOCP has received considerable attention from researchers because of its wide range of applications in many fields such as engineering technology, economic management, optimal control and design, machine learning, pattern recognition, combinatorial optimization, robust optimization, and so on (see, e.g., [1-8] and references therein).

SOCP includes linear programming (LP), convex quadratic programming (CQP) and quadratically constrained convex quadratic programming (QCCQP) as special cases. Furthermore, it is also a special case of semidefinite programming (SDP). Hence, SOCP problems can be solved theoretically by using a SDP method. Just like LP, CQP and SDP problems, SOCP problems can also be solved in polynomial time by interior-point methods (IPMs) [9]. However, the computational effort per iteration required by these methods to solve SOCP problems is greater than that required to solve LP and CQP problems but less than that required to solve SDP with the same size and structure. Solving SOCP by SDP approach is not advisable both on numerical grounds and computational complexity concerns. Thus, SOCP deserves to be researched in its own right, because of its computational tractability and its broad applicability. On the other hand, though smoothing Newton-type method can be used to solve SOCP problems, computing the exact solution to the system of equations is expensive when the problem is large-scale, and the solution may not be justified if initial point is far from the solution of the SOCP. However, Inexact method can overcome the drawbacks.

The standard SOCP problem is as follows

$$
\min \left\{\sum_{i=1}^{r}\left(c^{i}\right)^{T} x^{i}: \sum_{i=1}^{r} A_{i} x^{i}=b, x^{i} \in \mathrm{K}_{n_{i}}, i=1,2, \ldots, r\right\}
$$


where $A_{i} \in R^{m \times n_{i}}, c \in R^{n_{i}}$, and $b \in R^{m}$ are given data; $x^{i} \in \mathrm{K}_{n_{i}}$ are the variables; $\mathrm{K}_{n_{i}}$ is a second-order cone (SOC) with dimension $n_{i}$, which is defined by $\mathrm{K}_{n_{i}}=\left\{\left(x_{1} ; x_{2}\right) \mid x_{1} \in R, x_{2} \in R^{n_{i}-1}, x_{1} \geq\left\|x_{2}\right\|\right\} \subset R^{n_{i}}$. It is well known that $\mathrm{K}_{n_{i}}$ is a closed, pointed (i.e., $\mathrm{K}_{n_{i}} \cap\left(-\mathrm{K}_{n_{i}}\right)=\{0\}$ ) and convex cone. Hence SOCP problems are convex optimization problems. It is easy to verify that $\mathrm{K}_{n_{i}}$ is self-dual, that is, for each $x^{i} \in \mathrm{K}_{n_{i}}$, $\mathrm{K}_{n_{i}}=\mathrm{K}_{n_{i}}^{*}=\left\{s^{i} \in R^{n_{i}} \mid\left(s^{i}\right)^{T} x^{i} \geq 0\right\}$.

The dual problem of (1) is

$$
\max \left\{b^{T} y: A_{i}^{T} y+s^{i}=c^{i}, s^{i} \in \mathrm{K}_{n_{i}} i=1,2, \ldots, r\right\}
$$

where $y \in R^{m}$ is the variable and $s^{i} \in \mathrm{K}_{n_{i}}, i=1,2, \ldots, r$, are the slack variables. Denote $x=\left(x^{1} ; x^{2} ; \ldots ; x^{r}\right)$, where $x^{i} \in \mathrm{K}_{n_{i}}$. Correspondingly, define

$$
\begin{aligned}
& \mathrm{K}=\mathrm{K}_{n_{1}} \times \mathrm{K}_{n_{2}} \times \cdots \times \mathrm{K}_{n_{r}}, A=\left(A_{1}, A_{2}, \ldots, A_{r}\right) \in R^{m \times n}, \\
& c=\left(c^{1}, c^{2}, \ldots, c^{r}\right) \in R^{n}, s=\left(s^{1}, s^{2}, \ldots, s^{r}\right) \in R^{n},
\end{aligned}
$$

where $n_{1}+n_{2}+\ldots+n_{r}=n, r$ is the number of SOCs.

Problems (1) and (2) can be written as

$$
\min \left\{c^{T} x: A x=b, x \in \mathrm{K}\right\}
$$

and

$$
\max \left\{b^{T} y: A^{T} y+s=c, s \in \mathrm{K}, y \in R^{m}\right\}
$$

respectively, where $c \in R^{n}$ and $b \in R^{m}$. The vector $e=\left(e^{1} ; e^{2} ; \ldots ; e^{r}\right)$ is the identity element of $\mathrm{K}$, where $e^{i}=(1 ; 0) \in R \times R^{n_{i}-1}$ is the identity element of $\mathrm{K}_{n_{i}}$.

We refer to inequality $x \geq 0$ as the second-order cone inequality. For any $x \in R^{n}, x \geq 0$ if and only if $x^{T} s \geq 0$, for all $s \in \mathrm{K}$.

Throughout this paper, we make the following assumption.

ASSUMPTION 1.1 Both (3) and (4) are strictly feasible, i.e., there exists a vector $(x, y, s) \in \operatorname{int} \mathrm{K} \times R^{m} \times \operatorname{int} \mathrm{K}$ such that $A x=b$ and $A^{T} y+s=c$, where int $\mathrm{K}$ denotes the interior of $\mathrm{K}$.

It is well known that under Assumption 1.1, the $\operatorname{SOCP}(3)$ is equivalent to its optimality conditions: 


$$
\begin{aligned}
& A x=b, \\
& A^{T} y+s=c, \\
& x \circ s=0, x, s \in \mathrm{K}, y \in R^{m},
\end{aligned}
$$

where $x \circ s=0$ is referred to the complementary condition.

Interior-point method (IPM) is one of the most effective methods for solving SOCPs. Several IPMs designed for LP have been successfully extended to SOCP. There are extensive literatures focusing on IPMs for SOCPs $[5,7]$. IPMs typically deal with the following perturbation of the optimality conditions (5):

$$
\begin{aligned}
& A x=b, \\
& A^{T} y+s=c, \\
& x \circ s=\mu e, x, s \in \operatorname{int} \mathrm{K}, y \in R^{m},
\end{aligned}
$$

where $\mu>0$ is a parameter, and $e=(1 ; 0) \in R \times R^{n-1}$ is the identity element. This set of conditions are called the central path conditions as they define a trajectory approaching the solution set as $\mu \downarrow 0$. Conventional IPMs usually apply a Newton-type method to the equations in (6) with a suitable line search dealing with $x \in$ int $\mathrm{K}$ and $s \in$ int $\mathrm{K}$ explicitly.

It is proved that IPMs are globally convergent under certain assumptions. However, most IPMs need a feasible initial point with the exception of the infeasible IPMs (see, e.g., [10]). This is usually difficult in most cases.

Recently non-interior continuation methods have attracted much attention partially due to their superior numerical performances [11,12]. However, in order to prove their global/local-quadratic convergence, these algorithms either depend on the assumptions of uniform nonsingularity and strict complementarity or need to solve two linear systems of equations and perform at least two line searches at each iteration [13].

Motivated by this direction, the goal of the paper is to propose a new inexact non-interior continuation method for SOCP, which employs a new smoothing function to characterize the central path conditions. The new algorithm only needs to solve one system of linear equations inexactly and perform only one line search per iteration, and it can start from an arbitrary point. The global as well as the locally quadratic convergence of the proposed algorithm is analyzed, where the theory of Euclidean Jordan Algebra is used extensively.

The main difference of the non-interior continuation algorithm to be discussed in this paper from IPMs is that we reformulate (6) as a smoothing linear system of equations. It is shown that our algorithm has the following good properties:

(i) It can start from an arbitrary initial point;

(ii) It needs to solve only one linear system of equations inexactly and perform only one line search per iteration, and it can be fit for solve large-scale problems;

(iii) It is globally and locally Q-quadratically convergent under a mild assumption, without strict complementarity. The result is stronger than the many corresponding results of IPMs.

The following notations and terminologies are used throughout the paper. We use "," for adjoining vectors and matrices in a row and " $;$ " for adjoining them in a column. $R^{n}$ denotes the space of $n$-dimensional real 
column vectors, and $R^{n} \times R^{m}$ is identified with $R^{n+m}$. Denote $x^{2}=x \circ x \cdot \sqrt{x}$ is the unique vector in $R^{n}$ such that $x=\sqrt{x} \circ \sqrt{x}$.

For any $x, y \in R^{n}$, we write $x \geq y \quad(x>y)$ if $x-y \in \mathrm{K}(x-y \in \operatorname{int} \mathrm{K}) . R_{+}\left(R_{++}\right)$denotes the set of nonnegative (positive) real numbers.

This paper is organized as follows: In section 2, we give some preliminaries and the equivalent formulation of the perturbed optimality conditions. A smoothing function and its properties are given in section 3 . In section 4 , we describe an inexact non-interior continuation algorithm for SOCP. In section 5, the convergence of the the algorithm is analyzed. Numerical experiments are shown in section 6 . Some conclusions are given in section 7.

\section{Preliminaries and equivalent formulation of perturbed optimality conditions}

In this section, we mainly recall some basic results of Euclidean Jordan algebra, which are extensively used in this paper. The reader is referred to [13] for more details of Jordan algebra.

Let $J$ be a finite dimensional real vector space. $(J, \circ)$ is called a Jordan algebra if there exists a bilinear mapping $J \times J \rightarrow J$ denoted by "०" such that $x \circ y=y \circ x$ and $L_{x} L_{x^{2}}=L_{x^{2}} L_{x}$ for any $x, y \in J$, where $x^{2}=x \circ x, L_{x}: J \rightarrow J$ is a linear transformation defined by $L_{x} y:=x \circ y$.

A Jordan algebra has an identity, if there exists a unique element $e \in J$ such that $x \circ e=e \circ x=x$ for all $x \in J$. Let $(J, \circ)$ be a Jordan algebra. $(J, \circ)$ is called a Euclidean Jordan algebra if an associate inner product $\langle\cdot, \cdot\rangle$ is defined, and $\langle x \circ y, z\rangle=\langle x, y \circ z\rangle$ holds for any $x, y, z \in J$.

The set $\left\{x^{2}: x \in J\right\}$ is called the cone of squares of Euclidean Jordan algebra $(J, \circ,\langle\cdot\rangle$,$) . Let G(\mathrm{~K})$ denote the group of automorphisms of a cone $\mathrm{K}$. K is a homogeneous cone if $G(\mathrm{~K})$ acts on it transitively. That is, if $x, y \in$ int $\mathrm{K}$, then there exists $g \in G(\mathrm{~K})$ such that $g(x)=y$. Symmetric cones are cones that are self-dual and homogeneous. By Theorem III.2.1 in [13], we know that a cone is symmetric if and only if it is the cone of squares of some Euclidean Jordan algebra.

An element $c \in J$ is called idempotent if $c \circ c=c \neq 0$. Two elements $x, y \in J$ are orthogonal if $x \circ y=0$. An idempotent $c$ is primitive if it is nonzero and can't be expressed by sum of two other nonzero idempotents. For any $x \in J$, let $\operatorname{deg}(x)$ be the minimal positive integer such that $\left\{e, x, x^{2}, \ldots, x^{\operatorname{deg}(x)}\right\}$ is linearly dependent. Then rank of $J$, denoted by $r k(J)$, is defined as $\max \{\operatorname{deg}(x): x \in J\}$.

A set of primitive idempotents $\left\{c_{1}, c_{2}, \ldots, c_{r}\right\}$ is called a Jordan frame if $c_{i} \circ c_{j}=0$ for any $i, j \in\{1,2, \ldots, r\}$ with $i \neq j$ and $\sum_{i=1}^{r} c_{i}=e$, where $r k(J)=r$. 
THEOREM 2.1 (Spectral Decomposition Theorem [13]) Let $(J, \circ,\langle\cdot, \cdot\rangle)$ be a Euclidean Jordan algebra with $r k(J)=r$. Then for any $x \in J$, there exists a Jordan frame $\left\{c_{1}, c_{2}, \ldots, c_{r}\right\}$ and real numbers $\lambda_{1}, \lambda_{2}, \ldots, \lambda_{r}$ such that $x=\sum_{i=1}^{r} \lambda_{i} c_{i}$. The numbers $\lambda_{1}, \lambda_{2}, \ldots, \lambda_{r}$ are uniquely determined by $x$.

Every $\lambda_{i}(i=1,2, \ldots, r)$ is called an eigenvalue of $x$, which is a continuous function with respect to $x$ (see [14]). Define $\operatorname{tr}(x)=\sum_{i=1}^{r} \lambda_{i}$ and $\operatorname{det}(x)=\prod_{i=1}^{r} \lambda_{i}$, where $\operatorname{tr}(x)$ denotes the trace of $x$ and $\operatorname{det}(x)$ denotes the determinant of $x$.

For any $x, y \in J, x$ and $y$ are said to be operator commute if $L_{x}$ and $L_{y}$ commute, i.e., $L_{x} L_{y}=L_{y} L_{x}$. It is well known that $x$ and $y$ operator commute if and only if $x$ and $y$ have their spectral decompositions with respect to a common Jordan frame, i.e., $x=\sum_{i=1}^{r} \lambda_{i} c_{i}$ and $y=\sum_{i=1}^{r} w_{i} c_{i}$ for a Jordan frame $\left\{c_{1}, c_{2}, \ldots, c_{r}\right\}$.

We define the inner product $\langle\cdot, \cdot\rangle$ by $\langle x, y\rangle:=\operatorname{tr}(x \circ y)$ for any $x, y \in J$. Thus, we may define a norm on $J$ by

$$
\|x\|:=\sqrt{\langle x, x\rangle}=\sqrt{\operatorname{tr}\left(x^{2}\right)}=\sqrt{\sum_{i=1}^{r} \lambda_{i}^{2}}, \forall x \in J .
$$

An element $x \in J$ is said to be invertible if there exists some finite positive integer $k<\infty$ and some real numbers $\gamma_{i}$ such that the vector $y=\sum_{i=0}^{k} \gamma_{i} x^{i}$ satisfies $y \circ x=e$. We denote the inverse of $x$ as $x^{-1}$. If $x^{2}=y$ and $x \geq 0$, then $x$ can be written as $\sqrt{y}$. Given $x \in J$ with $x=\sum_{i=1}^{r} \lambda_{i} c_{i}$, where $\left\{c_{1}, c_{2}, \ldots, c_{r}\right\}$ is a Jordan frame and $\lambda_{1}, \lambda_{2}, \ldots, \lambda_{r}$ are eigenvalues of $x$, then $x^{2}=\sum_{i=1}^{r} \lambda_{i}^{2} c_{i}$. Furthermore, if $\lambda_{i} \geq 0$ for all $i \in\{1,2, \ldots, r\}$, then $x^{1 / 2}=\sum_{i=1}^{r} \lambda_{i}^{1 / 2} c_{i}$; if $\lambda_{i}>0$ for all $i \in\{1,2, \ldots, r\}$, then $x^{-1}=\sum_{i=1}^{r} \lambda_{i}^{-1} c_{i}$. More generally, we extend the definition of any real valued analytic function $g$ to elements of Euclidean Jordan algebras via their eigenvalues, i.e., $g(x)=\sum_{i=1}^{r} g\left(\lambda_{i}\right) c_{i}$ where $x \in J$ has the spectral decomposition $x=\sum_{i=1}^{r} \lambda_{i} c_{i}$.

Without loss of generality, in the following, we assume that $(J, \circ,\langle\cdot, \cdot\rangle)$ is a Euclidean Jordan algebra and $\mathrm{K}$ is its cone of squares, with $\langle u, v\rangle:=\operatorname{tr}(u \circ v)$ for any $u, v \in J$. Suppose that $(J, \circ,\langle\cdot, \cdot\rangle)$ has an identity element $e$ and $r k(J)=r=2$. Thus, instead of (1) and (2), we consider the following SOCP problem with a single SOC 
$\min _{x}\{\langle c, x\rangle: A x=b, x \in \mathrm{K}\}$

and its dual problem

$$
\max _{x}\left\{\langle b, y\rangle: A^{T} y+s=c, s \in \mathrm{K}, y \in R^{m}\right\}
$$

where $c \in R^{n}, A \in R^{m \times n}, b \in R^{m}$. Here and below, \|\|$\|$ refers to the standard Euclidean norm, and we denote $x=\left(x_{1} ; x_{2}\right)$ instead of $\left(x_{1}, x_{2}^{T}\right)^{T}$. For any $x=\left(x_{1} ; x_{2}\right), y=\left(y_{1} ; y_{2}\right) \in R \times R^{n-1}$, their Jordan product [13] is defined as $x \circ y=\left(x^{T} y ; x_{1} y_{2}+y_{1} x_{2}\right)$. It needs to note that our analysis can be easily extended to general cases with Cartesian product of SOCs.

For any vector $x=\left(x_{1} ; x_{2}\right) \in R \times R^{n-1}$, we define its spectral decomposition associated with SOC $\mathrm{K}$ as $x=\lambda_{1} u_{1}+\lambda_{2} u_{2}$

where the spectral values $\lambda_{i}$ and the associated spectral vectors $u_{i}$ of $x$ are given by

$$
\begin{aligned}
& \lambda_{i}=x_{1}+(-1)^{i}\left\|x_{2}\right\| \\
& u_{i}=\left\{\begin{array}{l}
\frac{1}{2}\left(1 ;(-1)^{i} \frac{x_{2}}{\left\|x_{2}\right\|}\right), x_{2} \neq 0 ; \\
\frac{1}{2}\left(1 ;(-1)^{i} w\right), x_{2}=0
\end{array}\right.
\end{aligned}
$$

For $i=1,2$, with any $w \in R^{n-1}$ such that $\|w\|=1$. If $x_{2} \neq 0$, then the decomposition (9) is unique. Some interesting properties of $\lambda_{1}, \lambda_{2}$ and $u_{1}, u_{2}$ are summarized below.

PROPERTY 2.1 For any $x=\left(x_{1} ; x_{2}\right) \in R \times R^{n-1}$, the spectral values $\lambda_{1}, \lambda_{2}$ and spectral vectors $u_{1}, u_{2}$ given by (10) and (11), have the following properties:

(i) $u_{1}+u_{2}=e$;

(ii) $u_{1}$ and $u_{2}$ are idempotent under the Jordan product, i.e., $u_{i}^{2}=u_{i}, i=1,2$;

(iii) $u_{1}$ and $u_{2}$ are orthogonal under the Jordan product and have length $\frac{\sqrt{2}}{2}$;

(iv) $\lambda_{1}, \lambda_{2}$ are nonnegative (respectively, positive) if and only if $x \in \mathrm{K}$ (respectively, $x \in \operatorname{int} \mathrm{K}$ ).

Given $x=\left(x_{1} ; x_{2}\right) \in R \times R^{n-1}$, the matrix of linear transformation is an arrow-shaped matrix defined by 
$L_{x}:=\left(\begin{array}{ll}x_{1} & x_{2}^{T} \\ x_{2} & x_{1} I\end{array}\right)$,

where $I$ represents the $(n-1) \times(n-1)$ identity matrix. It is easy to verify that $x \circ s=L_{x} s=L_{x} L_{s} e=L_{s} x=s \circ x$ for any $x, s \in R^{n}$. Moreover, $L_{x}$ is symmetric positive definite (semidefinite) if and only if $x \in$ int $\mathrm{K}(x \in \mathrm{K})$, i.e., $x>0(x \geq 0)$.

\section{A smoothing function and its properties}

In this section, we first introduce a new smoothing function. In [12], it has been shown that the vector-valued Fischer-Burmeister function $\varphi_{F B}(x, s): R^{n} \times R^{n} \rightarrow R^{n}$ defined by

$\varphi_{F B}(x, s)=x+s-\sqrt{x^{2}+s^{2}}$

satisfies the following important property

$\varphi_{F B}(x, s)=0 \Leftrightarrow x \geq 0, s \geq 0, x \circ s=0$.

The Fischer-Burmeister function has many interesting properties. However, it is typically nonsmooth, because it is not derivable at $(0,0) \in R \times R^{n-1}$. This undoubtedly limits its practical applications. Recently, some smoothing methods are presented, such as the method using Chen-Harker-Kanzow-Smale smoothing function [15].

In this paper, by smoothing the symmetric perturbed form of $\phi_{F B}$, we obtain a new vector-valued function $\Phi: R_{++} \times R^{n} \times R^{n} \rightarrow R^{n}$ as follows:

$$
\Phi(\mu, x, s)=x+s-\frac{1}{1+\mu}\left[(x+\mu s)^{2}+(\mu x+s)^{2}+2 \mu^{2}(1+\mu)^{2} e\right]^{\frac{1}{2}} .
$$

As we will show, the function $\Phi(\mu, x, s)$ has many nice properties which make it easy to characterize the central path conditions (6). In particular, $\Phi(\mu, x, s)$ is smooth for any $z:=(\mu, x, s) \in R_{++} \times R^{n} \times R^{n}$. This property plays an important role in the analysis of the quadratic convergence of our smoothing Newton method.

DEFINITION 3.1 [16] For a non-differentiable function $g: R^{n} \rightarrow R^{m}$, we consider a function $g_{\mu}: R^{n} \rightarrow R^{m}$ with a parameter $\mu>0$ that has the following properties:

(i) $g_{\mu}$ is differentiable for any $\mu>0$;

(ii) $\lim _{\mu \downarrow 0} g_{\mu}(x)=g(x)$ for any $x \in R^{n}$.

Such a function $g_{\mu}$ is called a smoothing function of $g$. 
In fact, we will prove in the following that the function $\Phi(\mu, x, s)$ given by (14) is a smoothing function of $\varphi_{F B}(x, s)$. Thus, we can solve a family of smoothing subproblems $\Phi(z)=0$ for $\mu>0$ and obtain a solution of $\varphi_{F B}(x, s)=0$ by letting $\mu \downarrow 0$.

Now, we give the main properties of $\Phi(\mu, x, s)$.

THEOREM 3.1 (i) $\Phi(\mu, x, s)$ is globally Lipschitz continuous for any $(\mu, x, s) \in R_{++} \times R^{n} \times R^{n}$. Moreover, $\Phi(\mu, x, s)$ is continuously differentiable at any $(\mu, x, s) \in R_{++} \times R^{n} \times R^{n}$ with its Jacobian given by

$\Phi^{\prime}(\mu, x, s)=\left(\begin{array}{c}L_{w}^{-1}\left[\frac{1}{(1+\mu)^{2}} L_{\left(w_{1}-w_{2}\right)}(x-s)-2 \mu e\right] \\ I-L_{w}^{-1}\left(\frac{1}{1+\mu} L_{w_{1}}+\frac{\mu}{1+\mu} L_{w_{2}}\right) \\ I-L_{w}^{-1}\left(\frac{\mu}{1+\mu} L_{w_{1}}+\frac{1}{1+\mu} L_{w_{2}}\right)\end{array}\right)$

where

$w_{1}:=\frac{1}{1+\mu} x+\frac{\mu}{1+\mu} s$

$w_{2}:=\frac{\mu}{1+\mu} x+\frac{1}{1+\mu} s$

$w:=\sqrt{w_{1}^{2}+w_{2}^{2}+2 \mu^{2} e}$,

(ii) $\lim _{\mu \downarrow 0} \Phi(\mu, x, s)=\varphi_{F B}(x, s)$ for any $(x, s) \in R^{n} \times R^{n}$. Thus, $\Phi(\mu, x, s)$ is a smoothing function of $\varphi_{F B}(x, s)$.

Proof. (i) It is not difficult to show that $\Phi(\mu, x, s)$ is globally Lipschitz continuous, and continuously differentiable at any $(\mu, x, s) \in R_{++} \times R^{n} \times R^{n}$. Now we prove (15). For any $z \in R_{++} \times R^{n} \times R^{n}$, from (18), we have

$w^{\prime}{ }_{\mu}=L_{w}^{-1}\left[L_{w_{1}}\left(w_{1}\right)^{\prime} \mu+L_{w_{2}}\left(w_{2}\right)^{\prime} \mu^{+2 \mu e] .}\right.$

It follows from (16) and (17) that

$\left(w_{1}\right)^{\prime} \mu=\frac{1}{(1+\mu)^{2}}(-x+s)$,

$\left(w_{2}\right)^{\prime}{ }_{\mu}=\frac{1}{(1+\mu)^{2}}(x-s)$. 
Thus, we obtain

$w^{\prime}{ }_{\mu}=L_{w}^{-1}\left[\frac{1}{(1+\mu)^{2}}\left(L_{w_{1}}-L_{w_{2}}\right)(-x+s)+2 \mu e\right]$.

Hence,

$\Phi^{\prime}{ }_{\mu}(z)=L_{w}^{-1}\left[\frac{1}{(1+\mu)^{2}} L_{\left(w_{1}-w_{2}\right)}(x-s)-2 \mu e\right]$.

Similarly, we can obtain

$w^{\prime}{ }_{x}=L_{w}^{-1}\left(\frac{1}{1+\mu} L_{w_{1}}+\frac{\mu}{1+\mu} L_{w_{2}}\right), \quad w^{\prime}{ }_{s}=L_{w}^{-1}\left(\frac{\mu}{1+\mu} L_{w_{1}}+\frac{1}{1+\mu} L_{w_{2}}\right)$.

Therefore, we have

$\Phi^{\prime}{ }_{x}(z)=I-L_{w}^{-1}\left(\frac{1}{1+\mu} L_{w_{1}}+\frac{\mu}{1+\mu} L_{w_{2}}\right)$

$\Phi^{\prime}{ }_{s}(z)=I-L_{w}^{-1}\left(\frac{\mu}{1+\mu} L_{w_{1}}+\frac{1}{1+\mu} L_{w_{2}}\right)$.

From (19)-(21), we obtain the desired result (15).

(ii) For any $x=\left(x_{1} ; x_{2}\right), s=\left(s_{1} ; s_{2}\right) \in R \times R^{n-1}$, it follows from the spectral factorization of $w^{2}=\lambda_{1}(\mu) u_{1}(\mu)+\lambda_{2}(\mu) u_{2}(\mu)$ that

$\Phi(z)=x+s-\left[\sqrt{\lambda_{1}(\mu)} u_{1}(\mu)+\sqrt{\lambda_{2}(\mu)} u_{2}(\mu)\right]$

where

$\lambda_{i}(\mu)=\left\|w_{1}\right\|^{2}+\left\|w_{2}\right\|^{2}+2 \mu^{2}+2(-1)^{i}\|v(\mu)\|, i=1,2$

$u_{i}(\mu)=\left\{\begin{array}{l}\frac{1}{2}\left(1 ;(-1)^{i} \frac{v(\mu)}{\|v(\mu)\|}\right), v(\mu) \neq 0 ; \\ \frac{1}{2}\left(1 ;(-1)^{i} w\right), v(\mu)=0,\end{array} i=1,2\right.$

$v(\mu)=\left(\frac{1}{1+\mu} x_{0}+\frac{\mu}{1+\mu} s_{0}\right)\left(\frac{\mu}{1+\mu} x_{1}+\frac{1}{1+\mu} s_{1}\right)+\left(\frac{\mu}{1+\mu} x_{0}+\frac{1}{1+\mu} s_{0}\right)\left(\frac{1}{1+\mu} x_{1}+\frac{\mu}{1+\mu} s_{1}\right)$,

with $w \in R^{n-1}$ being an arbitrary vector satisfying $\|w\|=1$. In a similar way, we can easily obtain 
$\varphi_{F B}(x, s)=x+s-\left(\sqrt{\lambda_{1}} u_{1}+\sqrt{\lambda_{2}} u_{2}\right)$,

where

$\lambda_{i}=\|x\|^{2}+\|s\|^{2}+2(-1)^{i}\|v\|, i=1,2$

$u_{i}=\left\{\begin{array}{l}\frac{1}{2}\left(1 ;(-1)^{i} \frac{v}{\|v\|}\right), v \neq 0 ; \\ \frac{1}{2}\left(1 ;(-1)^{i} w\right), v=0,\end{array} i=1,2\right.$

$v=x_{0} x_{1}+s_{0} s_{1}$

with $w \in R^{n-1}$ being the vector in $u_{i}(\mu)$. It is evident that $\lim _{\mu \downarrow 0} v(\mu)=v$. Hence,

$\lim _{\mu \downarrow 0} \lambda_{i}(\mu)=\lambda_{i, \mu} \lim _{\mu \downarrow 0} u_{i}(\mu)=u_{i, i}=1,2$.

Thus, we have $\lim _{\mu \downarrow 0} \Phi(\mu, x, s)=\varphi_{F B}(x, s)$. Therefore, it follows from (i) and Definition 3.1 that $\Phi(\mu, x, s)$ is a smoothing function of $\varphi_{F B}(x, s)$.

\section{The inexact non-interior continuation algorithm}

Based on the smoothing function (14) introduced in the previous section, the aim of this section is to propose a new inexact non-interior continuation algorithm for the SOCP and show its well-definedness under suitable assumptions.

Let $z:=(\mu, x, y)$. By using the smoothing function (14), we define the function $G(\mu, x, y): R_{++} \times R^{n} \times R^{m} \rightarrow R_{++} \times R^{m} \times R^{n}$ as follows:

$G(z):=\left(\begin{array}{c}\mu \\ A x-b \\ \Phi\left(\mu, x, c-A^{T} y\right)\end{array}\right)$.

In view of (13) and (22), $z^{*}:=\left(\mu^{*}, x^{*}, y^{*}\right)$ is a solution of the system $G(z)=0$ if and only if $\left(x^{*}, y^{*}, c-A^{T} y^{*}\right)$ solves the optimality conditions (5) [5,11]. It is well-known that problems (7) and (8) are equivalent to (22) in the sense that their solutions are coincident. Define merit function $\Psi: R_{+} \times R^{n} \rightarrow R_{+}$by

$\Psi(z):=\|G(z)\|^{2}$

Then, $z^{*}$ is a solution of $G(z)=0$ if and only if $\left(x^{*}, y^{*}, c-A^{T} y^{*}\right)$ is the optimal solution of (7) and (8). Therefore we can apply Newton's method to the nonlinear system of equations $G(z)=0$. 
Let $\gamma \in(0,1)$ and define the function $\beta(z)$ by

$$
\beta(z):=\gamma \min \left\{1,\|G(z)\|^{2}\right\}
$$

Next, we give the formal description of our algorithm.

ALGORITHM 4.1 (An inexact non-interior continuation algorithm for SOCP)

Step 0 Choose constants $\delta \in(0,1), \sigma \in(0,1)$, and $\mu_{0} \in R_{++}$, and let $\bar{z}:=\left(\mu_{0}, 0,0\right)$. Let $\left(x_{0}, y_{0}\right) \in R^{n} \times R^{m}$ be arbitrary initial point and $z_{0}:=\left(\mu_{0}, x_{0}, y_{0}\right)$. Choose $\gamma, t \in(0,1)$ such that $\gamma \mu_{0}<1 / 2,0 \leq t \leq 1-2 \gamma \bar{\mu}$. Let $\left\{t_{k}\right\}$ is a sequence such that $0 \leq t_{k} \leq t$. Set $k:=0$.

Step 1 If $G\left(z_{k}\right)=0$, then stop. Else, let

$$
\beta_{k}:=\beta\left(z_{k}\right):=\min \left\{\gamma, \gamma \Psi\left(z_{k}\right), \beta\left(z_{k-1}\right)\right\}
$$

Step 2 Compute $\Delta z_{k}:=\left(\Delta \mu_{k}, \Delta x_{k}, \Delta y_{k}\right)$ by solving the following system of linear equations

$G\left(z_{k}\right)+G^{\prime}\left(z_{k}\right) \Delta z_{k}=\left(\begin{array}{c}\bar{\mu} \beta\left(z_{k}\right) \\ r^{k}\end{array}\right)$

such that $\left\|r^{k}\right\| \leq t_{k}\left\|G\left(z_{k}\right)\right\|$, where $r^{k}=\Phi\left(z_{k}\right)+\Phi^{\prime}\left(z_{k}\right) \Delta z_{k}$.

Step 3 Let $\lambda_{k}=\max \left\{\delta^{v} \mid v=0,1,2, \ldots\right\}$ such that

$\left\|G\left(z_{k}+\delta^{v} \Delta z_{k}\right)\right\|^{2} \leq\left[1-\sigma\left(1-2 \gamma \bar{\mu}-t_{k}\right) \delta^{v}\right]\left\|G\left(z_{k}\right)\right\|^{2}$.

Let $\lambda_{k}:=\delta^{v_{k}}$

Step 4 Set $z_{k+1}:=z_{k}+\lambda_{k} \Delta z_{k}$ and $k:=k+1$. Go to step 1 .

To analyze Algorithm 4.1, we study the Lipschitzian, smoothness and differential properties of the function $G(z)$ given by (22). Moreover, we derive the $A$ computable formula for the Jacobian of the function $G(z)$ and give the condition for the Jacobian to be nonsingular. Throughout the rest of this paper, we make the following assumption:

ASSUMPTION 4.1 The matrix has full row rank.

LEMMA 4.1 [12] For any $x, s \in R^{n}$ and $w>_{\mathrm{K}} 0$, we have

$$
w^{2}>_{\mathrm{K}} x^{2}+s^{2} \Rightarrow L_{w}-L_{x}>0, L_{w}-L_{s}>0,\left(L_{w}-L_{x}\right)\left(L_{w}-L_{s}\right)>0 .
$$

Moreover, (29) remains true when " $>$ " is replaced by " $\geq$ ". 
THEOREM 4.1 Let $z:=(\mu, x, y)$ and $G: R_{++} \times R^{n} \times R^{m} \rightarrow R_{++} \times R^{m} \times R^{n}$ be defined by (22). Then the following results hold.

(i) $G$ is globally Lipschitz continuous, and continuously differentiable at any $z:=(\mu, x, y) \in R_{++} \times R_{n} \times R^{m}$ with its Jacobian given by

$$
G^{\prime}(z)=\left(\begin{array}{ccc}
1 & 0 & 0 \\
0 & A & 0 \\
B(z) & C(z) & -D(z) A^{T}
\end{array}\right)
$$

where

$$
\begin{aligned}
& B(z)=L_{\bar{w}}^{-1}\left[\frac{1}{(1+\mu)^{2}} L_{\left(\bar{w}_{1}-\bar{w}_{2}\right)}\left(x-c+A^{T} y\right)-2 \mu e\right] \\
& C(z)=I-L_{\bar{w}}^{-1}\left(\frac{1}{1+\mu} L_{\bar{w}_{1}}+\frac{\mu}{1+\mu} L_{\bar{w}_{2}}\right), \\
& D(z)=I-L_{\bar{w}}^{-1}\left(\frac{\mu}{1+\mu} L_{\bar{w}_{1}}+\frac{1}{1+\mu} L_{\bar{w}_{2}}\right) \\
& \bar{w}_{1}=\frac{1}{1+\mu} x+\frac{\mu}{1+\mu}\left(c-A^{T} y\right) \\
& \bar{w}_{2}=\frac{\mu}{1+\mu} x+\frac{1}{1+\mu}\left(c-A^{T} y\right) \\
& \bar{w}=\sqrt{\bar{w}_{1}^{2}+\bar{w}_{2}^{2}+2 \mu^{2} e} .
\end{aligned}
$$

(ii) Under Assumption 4.1, $G^{\prime}(z)$ is nonsingular for any $\mu>0$.

Proof. By Theorem 3.1, we can easily show that (i) holds. Now we prove (ii). For any fixed $\mu>0$, let $\Delta z:=(\Delta \mu, \Delta x, \Delta y) \in R \times R^{n} \times R^{m}$. It is sufficient to prove that the linear system of equations

$$
G^{\prime}(z) \Delta z=0
$$

has only zero solution, i.e., $\Delta \mu=0, \Delta x=0, \Delta y=0$. By (29) and (30), we have

$$
\begin{aligned}
& \Delta \mu=0, \\
& A \Delta x=0,
\end{aligned}
$$

$C(z) \Delta x-D(z) A^{T} \Delta y=0$. 
Premultiplying (33) by $L_{\bar{w}}$, we have

$$
\left(L_{\bar{w}}-\frac{1}{1+\mu} L_{\bar{w}_{1}}-\frac{\mu}{1+\mu} L_{\bar{w}_{2}}\right) \Delta x-\left(L_{\bar{w}}-\frac{\mu}{1+\mu} L_{\bar{w}_{1}}-\frac{1}{1+\mu} L_{\bar{w}_{2}}\right) A^{T} \Delta y=0 .
$$

On the other hand, since

$$
\bar{w}^{2}-\left(\bar{w}_{1}^{2}+\bar{w}_{2}^{2}\right)=\bar{w}^{2}-\left(\frac{1}{1+\mu} \bar{w}_{1}^{2}+\frac{\mu}{1+\mu} \bar{w}_{1}^{2}+\frac{\mu}{1+\mu} \bar{w}_{2}^{2}+\frac{1}{1+\mu} \bar{w}_{2}^{2}\right)=2 \mu^{2} e>_{\mathrm{K}} 0,
$$

it follows from Lemma 4.1 that

$$
L_{\bar{w}}-\frac{1}{1+\mu} L_{\bar{w}_{1}}-\frac{\mu}{1+\mu} L_{\bar{w}_{2}}>0
$$

$L_{\bar{w}}-\frac{\mu}{1+\mu} L_{\bar{w}_{1}}-\frac{1}{1+\mu} L_{\bar{w}_{2}}>0$

$\left(L_{\bar{w}}-\frac{1}{1+\mu} L_{\bar{w}_{1}}-\frac{\mu}{1+\mu} L_{\bar{w}_{2}}\right)\left(L_{\bar{w}}-\frac{\mu}{1+\mu} L_{\bar{w}_{1}}-\frac{1}{1+\mu} L_{\bar{w}_{2}}\right)>0$.

Hence $L_{\bar{w}}-\frac{\mu}{1+\mu} L_{\bar{w}_{1}}-\frac{1}{1+\mu} L_{\bar{w}_{2}}$ is invertible. Premultiplying (34) by

$$
\Delta x^{T}\left(L_{\bar{w}}-\frac{\mu}{1+\mu} L_{\bar{w}_{1}}-\frac{1}{1+\mu} L_{\bar{w}_{2}}\right)^{-1}
$$

and taking into account $A \Delta x=0$, we have

$$
\Delta x^{T}\left(L_{\bar{w}}-\frac{\mu}{1+\mu} L_{\bar{w}_{1}}-\frac{1}{1+\mu} L_{\bar{w}_{2}}\right)^{-1}\left(L_{\bar{w}}-\frac{1}{1+\mu} L_{\bar{w}_{1}}-\frac{\mu}{1+\mu} L_{\bar{w}_{2}}\right) \Delta x=0 .
$$

Denote

$$
\Delta \tilde{x}=\left(L_{\bar{w}}-\frac{2}{1+e^{\mu}} L_{\bar{w}_{1}}-\frac{e^{\mu}-1}{1+e^{\mu}} L_{\bar{w}_{2}}\right)^{-1} \Delta x .
$$

Then from (38), we obtain

$$
\Delta \tilde{x}^{T}\left(L_{\bar{w}}-\frac{1}{1+\mu} L_{\bar{w}_{1}}-\frac{\mu}{1+\mu} L_{\bar{w}_{2}}\right)\left(L_{\bar{w}}-\frac{\mu}{1+\mu} L_{\bar{w}_{1}}-\frac{1}{1+\mu} L_{\bar{w}_{2}}\right) \Delta \tilde{x}=0 .
$$


From (37) we know that $\left(L_{\bar{w}}-\frac{1}{1+\mu} L_{\bar{w}_{1}}-\frac{\mu}{1+\mu} L_{\bar{w}_{2}}\right)\left(L_{\bar{w}}-\frac{\mu}{1+\mu} L_{\bar{w}_{1}}-\frac{1}{1+\mu} L_{\bar{w}_{2}}\right)$ is positive definite. Therefore, it follows from (40) that $\Delta \tilde{x}=0$. Hence, (39) gives that $\Delta x=0$. Since $A$ has full row rank, (34) implies $\Delta y=0$. Thus the linear system of equations (30) has only zero solution, which shows that $G^{\prime}(z)$ is nonsingular. So we gain the required results.

\section{Convergence analysis}

In this section, we analyze the global and local convergence properties of Algorithm 4.1. It is shown that any accumulation point of the iteration sequence is a solution of the system $G(z)=0$. If the accumulation point $z$ * satisfies a nonsingularity assumption, then the iteration sequence converges to $z$ *locally Q-quadratically without strict complementarity. To show the global convergence of Algorithm 4.1, we need the following Lemma.

LEMMA 5.1 Suppose that Assumption 4.1 holds. For any $\tilde{z}:=(\tilde{\mu}, \tilde{x}, \tilde{y}) \in R_{++} \times R^{n} \times R^{m}$, if $G^{\prime}(\tilde{z})$ is nonsingular, then there exists a closed neighborhood $\mathrm{N}(\tilde{z})$ and a positive number $\bar{\alpha} \in(0,1]$ such that for any $z=(\mu, x, y) \in \mathrm{N}(\tilde{z})$ and all $\alpha \in[0, \bar{\alpha}]$, we have $\mu \in R_{++}, G^{\prime}(z)$ is invertible and

$\Psi(z+\alpha \Delta z) \leq\left[1-\sigma\left(1-2 \gamma \mu_{0}-t_{k}\right) \alpha\right] \Psi(z)$

Proof. Since $G^{\prime}(\tilde{z})$ is nonsingular and $\tilde{\mu}>0$, there exists a close neighborhood $\mathrm{N}(\tilde{z})$ of $\tilde{z}$ such that for any $z \in \mathrm{N}(\tilde{z})$, we have $\mu>0$ and that $G^{\prime}(z)$ is invertible. For any $z \in \mathrm{N}(\tilde{z})$, let $\Delta z=(\Delta \mu, \Delta x, \Delta y)$ be a unique solution of the following equation

$G(z)+G^{\prime}(z) \Delta z=\left(\begin{array}{c}\bar{\mu} \beta(z) \\ r\end{array}\right)$

where the residual vector $r=\Phi(z)+\Phi^{\prime}(z) \Delta z$ satisfies $\|r\| \leq t\|G(z)\|$. From (42), for any $z \in \mathrm{N}(\tilde{z})$ and all $\alpha \in[0,1]$, we have

$\mu+\alpha \Delta \mu=(1-\alpha) \mu+\alpha \bar{\mu} \beta(z)>0$.

When $\Psi(z)>1, \beta(z)=\gamma<\gamma \sqrt{\Psi(z)}=\gamma\|G(z)\|$, while $\Psi(z) \leq 1, \beta(z)=\gamma \Psi(z) \leq \gamma \sqrt{\Psi(z)}=\gamma\|G(z)\|$, thus for any $z \in R_{++} \times R^{n} \times R^{m}$

$\beta(z) \leq \gamma\|G(z)\|$

always holds. For any $\alpha \in[0,1]$, let

$r(\alpha):=G(z+\alpha \Delta z)-G(z)-\alpha G^{\prime}(z) \Delta z$,

Then by (42) and (44), we have

$$
\begin{aligned}
\|G(z)\| & \leq(1-\alpha)\|G(z)\|+\alpha \bar{\mu}\|\beta(z)\|+\alpha\|r\|+\|r(\alpha)\| \leq(1-\alpha)\|G(z)\|+\alpha \bar{\mu} \gamma\|G(z)\|+\alpha t_{k}\|G(z)\|+\|r(\alpha)\| \\
& \leq\left[1-\alpha\left(1-2 \gamma \mu_{0}-t_{k}\right)\right]\|G(z)\|+\|r(\alpha)\| .
\end{aligned}
$$


It is evident that $G(z)$ is continuously differentiable at $z \in \mathrm{N}(\tilde{z})$, which implies $\|r(\alpha)\|=o(\alpha)$. Therefore it follows (46) that there exists an $\bar{\alpha} \in(0,1)$ such that

$\|G(z+\alpha \Delta z)\| \leq\left[1-\sigma\left(1-2 \gamma \mu_{0}-t_{k}\right) \alpha\right]\|G(z)\|$

holds for all $\alpha \in(0, \bar{\alpha}]$ and all $z \in \mathrm{N}(\tilde{z})$. Thus, by (47), for all $\alpha \in(0, \bar{\alpha}]$ and all $z \in \mathrm{N}(\tilde{z})$, we have

$\Psi(z+\alpha \Delta z) \leq\left[1-\sigma\left(1-2 \gamma \mu_{0}-t_{k}\right) \alpha\right]^{2} \Psi(z) \leq\left[1-\sigma\left(1-2 \gamma \mu_{0}-t_{k}\right) \alpha\right] \Psi(z)$,

which is completed the proof.

THEOREM 5.1 Suppose that Assumption 4.1 holds and $\left\{z_{k}\right\}$ is the iteration sequence generated by Algorithm 4.1, then

(i) $\mu_{k} \in R_{++}$and $z_{k} \in \Xi$ for any $k \geq 0$, where

$\Xi=\left\{z \in R_{++} \times R^{n} \times R^{m}: \mu \geq \beta(z) \mu_{0}\right\}$

(ii) any accumulation point $z^{*}:=\left(\mu^{*}, x^{*}, y^{*}\right)$ of $\left\{z_{k}\right\}$ is a solution of $G(z)=0$.

Proof. (i) Suppose that $\mu_{k}>0$. It follows from (26) and Step 4 that

$$
\begin{aligned}
& \Delta \mu_{k}=-\mu_{k}+\beta(k) \mu_{0}, \\
& \mu_{k+1}=\mu_{k}+\lambda_{k} \Delta \mu_{k} .
\end{aligned}
$$

Substituting (49) into (50), we have

$$
\mu_{k+1}=\mu_{k}-\lambda_{k} \mu_{k}+\lambda_{k} \beta_{k} \mu_{0}=\left(1-\lambda_{k}\right) \mu_{k}+\lambda_{k} \beta_{k} \mu_{0}>0
$$

which, together with $\mu_{0}>0$ and $\lambda_{k}=\delta_{k}^{v_{k}} \in(0,1)$ implies that $\mu_{k} \in R_{++}$for any $k \geq 0$.

Now we prove $z_{k} \in \Xi$ for any $k \geq 0$ by induction. Since $\beta_{0}=\beta\left(z_{0}\right)=\gamma \min \left\{1,\left\|G\left(z_{0}\right)\right\|^{2}\right\} \leq \gamma \in(0,1)$, it is easy to see that $z_{0} \in \Xi$. Suppose that $z_{k} \in \Xi$, then

$$
\mu_{k} \geq \beta_{k} \mu_{0}
$$

We consider the following two cases:

Case (I): If $\left\|G\left(z_{k}\right)\right\|>1$, then

$\beta_{k}=\gamma$.

Since $\beta_{k+1}=\gamma \min \left\{1,\left\|G\left(z_{k+1}\right)\right\|^{2}\right\} \leq \gamma$, it follows from (27), (43), (52) and (53) that 
$\mu_{k+1}-\beta_{k+1} \mu_{0} \geq\left(1-\lambda_{k}\right) \beta_{k} \mu_{0}+\lambda_{k} \beta_{k} \mu_{0}-\gamma \mu_{0}=\beta_{k} \mu_{0}-\gamma \mu_{0}=0$

Case (II): If $\left\|G\left(z_{k}\right)\right\| \leq 1$, then

$\beta_{k}=\gamma\left\|G\left(z_{k}\right)\right\|^{2}$

By (27), $\left\|G\left(z_{k+1}\right)\right\| \leq\left\|G\left(z_{k}\right)\right\| \leq 1$. From (43), (55), and taking into account $\beta_{k+1}=\gamma\left\|G\left(z_{k+1}\right)\right\|^{2}$, we have

$$
\begin{aligned}
\mu_{k+1}-\beta_{k+1} \mu_{0} & =\left(1-\lambda_{k}\right) \mu_{k}+\lambda_{k} \beta_{k} \mu_{0}-\gamma \mu_{0}\left\|G\left(z_{k+1}\right)\right\|^{2} \geq\left(1-\lambda_{k}\right) \beta_{k} \mu_{0}+\lambda_{k} \beta_{k} \mu_{0}-\gamma \mu_{0}\left\|G\left(z_{k}\right)\right\|^{2} \\
& =\beta_{k} \mu_{0}-\gamma \mu_{0}\left\|G\left(z_{k}\right)\right\|^{2}=0 .
\end{aligned}
$$

Combining (54) and (56) yields that $z_{k} \in \Xi$ for any $k \geq 0$.

Next, we prove (ii). Without loss of generality, we assume that $\left\{z_{k}\right\}$ converges to $z^{*}$ as $k \rightarrow+\infty$. Since $\left\{\left\|G\left(z_{k}\right)\right\|\right\}$ is monotonically decreasing and bounded from below, it follows from the continuity of $G(\cdot)$ that $\left\{\left\|G\left(z_{k}\right)\right\|\right\}$ converges to a nonnegative number $G\left(z^{*}\right)$. Then by the definition of $\beta(\cdot)$, we obtain that $\left\{\beta_{k}\right\}$ converges to $\beta^{*}=\gamma \min \left\{1,\left\|G\left(z^{*}\right)\right\|^{2}\right\}$. On account of (43) and Theorem 5.1 (i), we have

$0<\mu_{k+1}=\left(1-\lambda_{k}\right) \mu_{k}+\lambda_{k} \beta_{k} \mu_{0} \leq \mu_{k}$,

which implies that $\left\{\mu_{k}\right\}$ converges to $\mu^{*}$. If $\left\|G\left(z^{*}\right)\right\|=0$, then we obtain the desired result. In the following, we suppose $\left\|G\left(z^{*}\right)\right\|>0$. By Lemma 4.1, $0<\beta^{*} \mu_{0} \leq \mu^{*}$. It follows from Theorem 4.1 that $G^{\prime}\left(z^{*}\right)$ exists and it is invertible. Hence, by Lemma 5.1, there exists a closed neighborhood $\mathrm{N}(\tilde{z})$ of $\tilde{z}$ and a positive number $\bar{\alpha} \in(0,1]$ such that for any $z=(\mu, x, y) \in \mathrm{N}(\tilde{z})$ and $\alpha \in[0, \bar{\alpha}]$, we have $\mu \in R_{++}, G^{\prime}(z)$ is invertible and

$\|G(z+\alpha \Delta z)\|^{2} \leq\left[1-\sigma\left(1-2 \gamma \mu_{0}-t_{k}\right) \alpha\right]\|G(z)\|^{2}$.

Therefore, for a nonnegative integer $\bar{v}$ such that $\delta^{\bar{\nu}} \in(0, \bar{\alpha}]$, and all sufficiently large $k$, we have $\left\|G\left(z+\delta^{\bar{v}} \Delta z\right)\right\|^{2} \leq\left[1-\sigma\left(1-2 \gamma \mu_{0}-t_{k}\right) \delta^{\bar{v}}\right]\|G(z)\|^{2}$

Note that for all $k$ large enough, $\lambda_{k}=\delta^{v_{k}} \geq \delta^{\bar{v}}$, it follows from (27) that $\left\|G\left(z+\delta^{\bar{v}} \Delta z\right)\right\|^{2} \leq\left[1-\sigma\left(1-2 \gamma \mu_{0}-t_{k}\right) \lambda_{k}\right]\|G(z)\|^{2} \leq\left[1-\sigma\left(1-2 \gamma \mu_{0}-t_{k}\right) \delta^{\bar{v}}\right]\|G(z)\|^{2}$

This contradicts the fact that the sequence $\left\{\left\|G\left(z_{k}\right)\right\|\right\}$ converges to $\left\|G\left(z^{*}\right)\right\|>0$. This completes the proof. To establish the locally Q-quadratic convergence of Algorithm 4.1, we need the following assumption: 
ASSUMPTION 5.1 Assume that $z^{*}$ satisfies the nonsingularity condition, i.e., all $V \in \partial G\left(z^{*}\right)$ are nonsingular. Now we are in the position to give the local convergence result for Algorithm 4.1.

THEOREM 5.2 Suppose that Assumption 4.1 holds and that $z^{*}$ is an accumulation point of the iteration sequence $\left\{z_{k}\right\}$ generated by Algorithm 4.1. If Assumption 5.1 holds, then

(i) $\lambda_{k} \equiv 1$ for all $z_{k}$ sufficiently close to $z^{*}$;

(ii) $\left\{z_{k}\right\}$ converges to $z^{*}$ Q-quadratically, i.e., $\left\|z_{k+1}-z^{*}\right\|=O\left(\left\|z_{k}-z^{*}\right\|^{2}\right)$. Moreover, $\mu_{k+1}=O\left(\mu_{k}^{2}\right)$.

Proof. By using Theorem 4.1, we can prove the theorem similarly as Theorem 8 in [11]. For brevity, we omit the details here.

It should be noted that the local Q-quadratic convergence of Algorithm 4.1 depends on the strong semismoothness of the new smoothing function.

\section{Numerical experiments}

In this section, we conducted some numerical experiments to evaluate the efficiency of Algorithm 4.1. All these experiments were performed on an Lenovo notebook computer Y470 with Intel(R) Core(TM) i7-2630QM CPU $2.00 \mathrm{GHz}$ and $6 \mathrm{~GB}$ memory. The operating system was Windows WIN 7 and the implementations were done in MATLAB 7.0.1.

In all these experiments, we choose

$$
x_{0}=e=(1 ; 0) \in R \times R^{n-1}, y_{0}=0 \in R^{m}
$$

as initial points. The parameters used in Algorithm 4.1 were as follows:

$\mu_{0}=0.001, \sigma=0.25, \delta=0.65, \gamma=0.85$.

We used $\|G(z)\| \leq 10^{-10}$ as the stopping criterion.

Firstly, for given size $m$ and $n$, we randomly generate six test problems. To be specific, we generate a random matrix $A \in R^{m \times n}$ with full row rank and random vectors $x \in \operatorname{int} \mathrm{K}, s \in \operatorname{int} \mathrm{K}, y \in R^{m}$, and then let $b:=A x$ and $c:=A^{T} y+s$. Thus the generated SOCP problems and their corresponding dual problems have optimal solutions and their optimal values coincide, since the set of strictly feasible solutions of SOCP and its dual problem are nonempty. For comparison purpose, we also use SDPT3 [17] to solve the same problems.

The results are listed in Table 1 which indicates that Algorithm 4.1 performs very well. We also obtained similar results for other random examples.

Table 1. Comparison of Algorithm 4.1 and SDPT3 for randomly generated SOCP problems

\begin{tabular}{|l|l|l|l|l|l|l|}
\hline & & \multicolumn{3}{|l|}{ SDPT3 } & \multicolumn{2}{l|}{ Algorithm 4.1 } \\
\hline problem & $m$ & $n$ & IT & CPU(s) & IT & CPU(s) \\
\hline
\end{tabular}




\begin{tabular}{|l|l|l|l|l|l|l|} 
problem 1 & 50 & 50 & 7 & 0.12 & 5 & 0.10 \\
\hline problem 2 & 100 & 100 & 10 & 0.19 & 7 & 0.13 \\
\hline problem 3 & 150 & 150 & 16 & 0.38 & 12 & 0.31 \\
\hline problem 4 & 200 & 200 & 20 & 1.25 & 14 & 0.86 \\
\hline problem 5 & 250 & 250 & 27 & 1.96 & 19 & 1.27 \\
\hline problem 6 & 300 & 300 & 38 & 2.31 & 26 & 1.42 \\
\hline
\end{tabular}

Next, we consider the following SOCP problem $\min \left\{\langle c, x\rangle: A x=b, x \in \mathrm{K}_{n}\right\}$, whose data are given as follows:

$A=\left(\begin{array}{ccccc}50 & 6 & & & \\ -3 & 50 & 6 & & \\ & \ddots & \ddots & \ddots & \\ & & -3 & 50 & 6 \\ & & & -3 & 50\end{array}\right) \in R^{m \times n}$

$c=25 e+2 \operatorname{rand}(n, 1)-\operatorname{ones}(n, 1)$,

$b=25 e+2 \operatorname{rand}(m, 1)-$ ones $(m, 1)$

where ones $(k, 1)$ denotes the vector with dimension $k$ and its elements being all ones. Note that the problem has representativeness to some extent.

Table 2 displays the numerical results of Algorithm 4.1 for the above problem. The present method is efficient as far as the numerical results are considered. Algorithm 4.1 can also deal with large-scale and sparse secondorder cone programming efficiently. Therefore, the new method may be of practical interest.

Table 2. Numerical results for Algorithm 4.1

\begin{tabular}{lllll}
\hline$m$ & $n$ & Iter & CPU time $(\mathrm{s})$ & $\|G(z)\|$ \\
\hline 50 & 50 & 6 & 0.1220 & $3.152479 \times 10^{-13}$ \\
100 & 100 & 7 & 0.4771 & $4.901354 \times 10^{-13}$ \\
150 & 150 & 7 & 1.7603 & $2.572183 \times 10^{-13}$ \\
200 & 200 & 8 & 2.0476 & $5.349761 \times 10^{-13}$ \\
250 & 250 & 9 & 2.1742 & $7.026815 \times 10^{-13}$ \\
300 & 300 & 10 & 1.8394 & $3.572470 \times 10^{-13}$ \\
350 & 350 & 10 & 2.5328 & $7.478249 \times 10^{-13}$ \\
400 & 400 & 9 & 2.1761 & $8.152840 \times 10^{-13}$ \\
450 & 450 & 10 & 2.9637 & $6.33262 \times 10^{-13}$ \\
500 & 500 & 11 & 3.7648 & $5.467159 \times 10^{-13}$ \\
\hline
\end{tabular}




\section{Conclusions}

Based on a new smoothing function, we give a new inexact non-interior continuation algorithm for SOCP problems. The presented algorithm solves only one system of linear equations inexactly and performs only one line search at each iteration, and it can start from an arbitrary point. The algorithm is globally and locally Qquadratically convergent under a mild assumption without strict complementarity. The result is stronger than that of the corresponding results of IPMs.

\section{Acknowledgment}

The work is supported by Project of Natural Science Foundation of Shandong province (ZR2016AM06).

\section{References}

1. Liu, D., Li, L. \& Chen, X., Pattern synthesis of a practical conformal hydrophone array via second-order cone programming, Cluster Comput (2018), 1-18. https://doi.org/10.1007/s10586-018-1836-5.

2. Kanno, Y. \& Yamada, H., A note on truss topology optimization under self-weight load: mixed-integer second-order cone programming approach, Structural and Multidisciplinary Optimization, 56(1): 221-226, 2017.

3. Ukritchon, B. \& Keawsawasvong, S., Stability of Retained Soils Behind Underground Walls with an Opening Using Lower Bound Limit Analysis and Second-Order Cone Programming,1-17, 2018 Geotechnical and Geological Engineering, https://doi.org/10.1007/s10706-018-0710-9.

4. Zhang, Y., A projected-based neural network method for second-order cone programming, International Journal of Machine Learning and Cybernetics, 8(6):1907-1914, 2017.

5. F. Alizadeh, D. Goldfarb, Second-order cone programming, Mathematical Programming, 95(1)(2003), 3-51.

6. M.S. Lobo, L. Vandenberghe, S. Boyd, H. Lebret, Applications of second-order cone programming, Linear Algebra and its Applications, 284(1-3)(1998), 193-228.

7. S.H. Schmieta, F. Alizadeh, Extension of primal-dual interior point algorithms to symmetric cones, Mathematical Programming, Series A., 96(3)(2003), 409-438.

8. P. Tseng, Second-order cone programming relaxation of sensor network localization, SIAM Journal on Optimization, 18(1)(2007), 156-185.

9. R.D.C. Monteiro, T. Tsuchiya, Polynomial Convergence of Primal-Dual Algorithms for the Second-Order Cone Program Based on the MZ-Family of Directions, Mathematical Programming, 88(1)(2000), 61-83.

10. B.K. Rangarajan, Polynomial Convergence of Infeasible-Interior-Point Methods over Symmetric Cones, SIAM J. OPTIM., 16(4)(2006), 1211-1229.

11. J. Faraut, A. Koranyi, Analysis on symmetric cones, Oxford University Press, London and New York, 1994.

12. M. Fukushima, Z. Luo, P. Tseng, Smoothing functions for second order cone complimentarity problems, SIAM J. Optim., 2(2)(2001), 436-460.

13. J. Faraut, A. Koranyi, Analysis on symmetric cones, Oxford University Press, London and New York, 1994.

14. M.S. Gowda, R. Sznajder, J. Tao, Some P-properties for linear transformations on Euclidean Jordan algebras, Linear Algebra Appl., 393(2004), 203-232.

15. L. Qi, J. Sun, A nonsmooth version of Newton's method, Mathematical Programming, 58(1-3)(1993), 353367.

16. S. Hayashi, N. Yamashita, M. Fukushima, A combined smoothing and regularized method for monotone second-order cone complementarity problems, SIAM Journal on Optimization, 15(2)(2005), 593-615. 
17. K.C. Toh, R.H. Tütüncü, M.J. Todd, On the implementation of SDPT3 (version 3.1) - a Matlab software package for semidefinite-quadratic-linear programming, Invited Paper, 2004 IEEE Conference on Computer-Aided Control System Design, Taipei, Taiwan.

\section{Authors' biography with Photo}

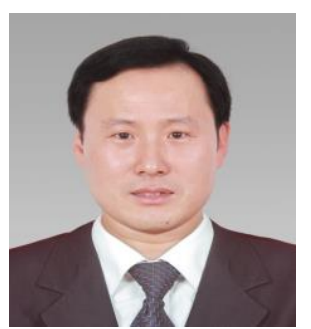

Liang Fang is a professor at Taishan University. He obtained his PhD from Shanghai Jiaotong University in June, 2010. His research interests are in the areas of cone optimizations and complementarity problems. 